\title{
Oscillatory behaviour of solutions of forced neutral differential equations
}

\author{
by N. Parhi and P. K. Mohanty (Berhampur)
}

\begin{abstract}
Sufficient conditions are obtained for oscillation of all solutions of a class of forced $n$th order linear and nonlinear neutral delay differential equations. Also, asymptotic behaviour of nonoscillatory solutions of a class of forced first order neutral equations is studied.
\end{abstract}

1. This paper is concerned with oscillatory behaviour of solutions of forced neutral delay differential equations (NDDE) of the form

$$
\left[x(t)+\sum_{i=1}^{l} p_{i}(t) x\left(t-\tau_{i}\right)\right]^{(n)}+\delta \sum_{j=1}^{m} q_{j}(t) x\left(t-\sigma_{j}\right)=f(t)
$$

and

$$
\left[x(t)+\sum_{i=1}^{l} p_{i}(t) g_{i}\left(x\left(t-\tau_{i}\right)\right)\right]^{(n)}+\delta \sum_{j=1}^{m} q_{j}(t) h_{j}\left(x\left(t-\sigma_{j}\right)\right)=f(t),
$$

where $p_{i}, q_{j}, f \in C\left(\left[t_{0}, \infty\right), \mathbb{R}\right)$ and $g_{i}, h_{j} \in C(\mathbb{R}, \mathbb{R})$ are such that $p_{i}(t) \geq 0$, $q_{j}(t) \geq 0, x g_{i}(x)>0$ for $x \neq 0, x h_{j}(x)>0$ for $x \neq 0, \tau_{i} \geq 0$ and $\sigma_{j} \geq 0$ for $i=1, \ldots, l$ and $j=1, \ldots, m$.

Let $\phi \in C\left(\left[t_{0}-\varrho, t_{0}\right], \mathbb{R}\right)$, where $\varrho=\max \left\{\tau_{i}, \sigma_{j} \mid i=1, \ldots, l\right.$ and $j=$ $1, \ldots, m\}$. By a solution of (1.2) on $\left[t_{0}, \infty\right)$ with initial function $\phi$ we mean a function $x \in C\left(\left[t_{0}-\varrho, \infty\right), \mathbb{R}\right)$ such that $x(t)=\phi(t)$ for $t \in\left[t_{0}-\varrho, t_{0}\right]$, $x(t)+\sum_{i=1}^{l} p_{i}(t) g_{i}\left(x\left(t-\tau_{i}\right)\right)$ is $n$ times continuously differentiable for $t \geq t_{0}$ and $x(t)$ satisfies (1.2) for $t \geq t_{0}$. Such a solution is said to be oscillatory if it has arbitrarily large zeros; otherwise, it is said to be nonoscillatory. These statements also hold good for the equation (1.1).

1991 Mathematics Subject Classification: 34C10, 34K15, 34K40.

Key words and phrases: neutral equation, oscillation, asymptotic behaviour, forced equation.

This work has been done under a scheme supported by Berhampur University grant Nos. 13518/P and R/BU/90. 
In recent years there has been a growing interest in oscillation theory of NDDE. However, most of the literature is concerned with linear homogeneous equations (see for example $[1,2,3,6,7]$ and the references therein). Some authors $[4,5]$ have considered the nonlinear NDDE of the form

$$
[x(t)+p(t) x(t-\tau)]^{(n)}+f(t, x(t-\sigma))=0 .
$$

But their conditions are such that the results they have obtained are not applicable to the equations considered here.

2. In this section we study the oscillatory behaviour of solutions of (1.1) and (1.2).

Theorem 1. Suppose that each $p_{i}(t)$ is bounded and for some $j=k$, $q_{k}(t) \neq 0$ in any neighbourhood of infinity and $q_{k}(t)$ is $\tau$-periodic, where $N_{i} \tau=\tau_{i}$ and the $N_{i}$ 's are positive integers. Further, assume that

$\left(\mathrm{A}_{1}\right) \quad$ there exists a function $F \in C^{n}\left(\left[t_{0}-\sigma, \infty\right), \mathbb{R}\right)$ such that $F^{(n)}(t)=$ $f(t)$ for $t \geq t_{0}$, and

$\left(\mathrm{A}_{2}\right) \quad \int_{t_{0}}^{\infty} q_{k}(t) F_{ \pm}\left(t-\sigma_{k}\right) d t=\infty$

where $\sigma=\max \left\{\sigma_{1}, \ldots, \sigma_{m}\right\}, F_{+}(t)=\max \{F(t), 0\}, F_{-}(t)=\max \{-F(t), 0\}$. Then (a) all solutions of (1.1) oscillate for $\delta=1$, and (b) all bounded solutions of (1.1) oscillate for $\delta=-1$ and bounded $F(t)$.

Proof. Assume on the contrary that $x(t)$ is a nonoscillatory solution of (1.1). Let $x(t)>0$ ultimately. The case $x(t)<0$ for large $t$ may be treated similarly. So there exists a $t_{1}>t_{0}$ such that $x(t)>0, x\left(t-\tau_{i}\right)>0$ and $x\left(t-\sigma_{j}\right)>0$ for $t \geq t_{1}, i=1, \ldots, l$ and $j=1, \ldots, m$. Setting, for $t \geq t_{1}$,

$$
z(t)=x(t)+\sum_{i=1}^{l} p_{i}(t) x\left(t-\tau_{i}\right)-F(t)
$$

we obtain

$$
z^{(n)}(t)=-\delta \sum_{j=1}^{m} q_{j}(t) x\left(t-\sigma_{j}\right) .
$$

Consequently, $z^{(r)}(t)>0$ or $<0$ for large $t$, and $r=0,1, \ldots, n-1$. If $z(t)<0$ for $t \geq t_{2}>t_{1}$, then by (2.1), $F(t)>0$ and hence

$$
\begin{aligned}
\int_{t_{0}}^{\infty} q_{k}(t) F_{-}\left(t-\sigma_{k}\right) d t & =\int_{t_{0}}^{t_{2}+\sigma_{k}} q_{k}(t) F_{-}\left(t-\sigma_{k}\right) d t+\int_{t_{2}+\sigma_{k}}^{\infty} q_{k}(t) F_{-}\left(t-\sigma_{k}\right) d t \\
& =\int_{t_{0}}^{t_{2}+\sigma_{k}} q_{k}(t) F\left(t-\sigma_{k}\right) d t<\infty
\end{aligned}
$$


a contradiction to $\left(\mathrm{A}_{2}\right)$. So $z(t)>0$ for $t \geq t_{2}$ and hence

$$
F_{+}(t)<x(t)+\sum_{i=1}^{l} p_{i}(t) x\left(t-\tau_{i}\right) .
$$

Let $\delta=1$. Then $z^{(n)}(t) \leq-q_{k}(t) x\left(t-\sigma_{k}\right)$ for $t \geq t_{2}$. Clearly, $z^{(n-1)}(t)>$ 0 for large $t$; otherwise $z(t)<0$ for large $t$, a contradiction. So integration of (2.2) for $t \geq t_{3}>t_{2}+\sigma_{k}$ yields

$$
\int_{t_{3}}^{\infty} q_{k}(t) x\left(t-\sigma_{k}\right) d t \leq z^{(n-1)}\left(t_{3}\right)<\infty .
$$

Moreover, for each $i$ and for $t \geq t_{4}>t_{3}+\max \left\{\tau_{1}, \ldots, \tau_{l}\right\}$,

$$
\begin{aligned}
& \int_{t_{4}}^{\infty} p_{i}\left(t-\sigma_{k}\right) q_{k}(t) x\left(t-\tau_{i}-\sigma_{k}\right) d t \\
& \quad \leq L \int_{t_{4}-\tau_{i}}^{\infty} q_{k}\left(t+\tau_{i}\right) x\left(t-\sigma_{k}\right) d t \leq L \int_{t_{4}-\tau_{i}}^{\infty} q_{k}(t) x\left(t-\sigma_{k}\right) d t<\infty,
\end{aligned}
$$

where $L>0$ is the bound of each $p_{i}(t)$. Consequently,

$$
\begin{aligned}
\int_{t_{0}}^{\infty} q_{k}(t) F_{+}\left(t-\sigma_{k}\right) d t \leq & \int_{t_{0}}^{t_{4}} q_{k}(t) F_{+}\left(t-\sigma_{k}\right) d t \\
& +\int_{t_{4}}^{\infty} q_{k}(t) x\left(t-\sigma_{k}\right) d t \\
& +\sum_{i=1}^{l} \int_{t_{4}}^{\infty} q_{k}(t) p_{i}\left(t-\sigma_{k}\right) x\left(t-\sigma_{k}-\tau_{i}\right) d t
\end{aligned}
$$

in view of (2.3) and (2.4). This contradiction completes the proof in case $\delta=1$.

Let $\delta=-1$ and $F(t)$ be bounded. In this case, for $t \geq t_{1},(2.2)$ gives $z^{(n)}(t) \geq q_{k}(t) x\left(t-\sigma_{k}\right) \geq 0$. If $x(t)$ is bounded, then so is $z(t)$, and since $z^{(n-1)}(t)$ is strictly increasing, it is bounded. Therefore,

$$
\int_{t_{3}}^{\infty} q_{k}(t) x\left(t-\sigma_{k}\right) d t<\infty
$$

and hence the inequality (2.4) holds. Thus the required contradiction follows from (2.4), (2.5) and (2.6).

Hence the theorem is proved.

The following example shows that the conditions of Theorem 1(b) are not sufficient for all solutions of (1.1) with $\delta=-1$ to be oscillatory. 
EXAmPle 1. Consider the equation

$$
[x(t)+x(t-\pi)]^{\prime}-e^{\pi / 2}\left(1+e^{-\pi}\right) x(t-\pi / 2)=e^{\pi / 2}\left(1+e^{-\pi}\right) \cos t
$$

for $t \geq \pi$. Here all the conditions of Theorem 1(b) are satisfied, with $F(t)=$ $e^{\pi / 2}\left(1+e^{-\pi}\right) \sin t$, but (2.7) admits an unbounded nonoscillatory solution $x(t)=e^{t}+\sin t$.

Theorem 2. Assume that $\left(\mathrm{A}_{1}\right)$ is satisfied and

$$
\liminf _{t \rightarrow \infty}\left[F(t) / t^{n-1}\right]=-\infty \quad \text { and } \quad \limsup _{t \rightarrow \infty}\left[F(t) / t^{n-1}\right]=\infty .
$$

Then (a) all solutions of (1.2) oscillate for $\delta=1$, and (b) all bounded solutions of (1.2) oscillate for $\delta=-1$ and bounded $p_{i}(t)$.

Proof. Suppose that $x(t)$ is an eventually positive solution of (1.2). Parallel arguments hold when $x(t)<0$ eventually. Then $x(t)>0, x\left(t-\tau_{i}\right)>$ 0 and $x\left(t-\sigma_{j}\right)>0$ for $t \geq t_{1}>\max \left\{t_{0}, 0\right\}, i=1, \ldots, l$ and $j=1, \ldots, m$. We set, for $t \geq t_{1}$,

$$
z(t)=x(t)+\sum_{i=1}^{l} p_{i}(t) g_{i}\left(x\left(t-\tau_{i}\right)\right)>0 .
$$

Hence the equation (1.2) yields

$$
z^{(n)}(t)=f(t)-\delta \sum_{j=1}^{m} q_{j}(t) h_{j}\left(x\left(t-\sigma_{j}\right)\right) .
$$

Let $\delta=1$. Then $z^{(n)}(t) \leq f(t)$ for $t \geq t_{1}$. Repeated integration from $t_{1}$ to $t$ of this inequality gives

$$
z(t) \leq \mu_{n}+\mu_{n-1}\left(t-t_{1}\right)+\ldots+\frac{\mu_{1}}{(n-1) !}\left(t-t_{1}\right)^{n-1}+F(t),
$$

where $\mu_{1}, \ldots, \mu_{n}$ are constants. Therefore, for $t \geq t_{1}$,

$$
\frac{z(t)}{t^{n-1}} \leq \mu_{n} \frac{1}{t^{n-1}}+\mu_{n-1} \frac{\left(1-t_{1} / t\right)}{t^{n-1}}+\ldots+\mu_{1} \frac{\left(1-t_{1} / t\right)^{n-1}}{(n-1) !}+\frac{F(t)}{t^{n-1}} .
$$

Using the first condition of $\left(\mathrm{A}_{3}\right)$, it follows that

$$
0 \leq \liminf _{t \rightarrow \infty} z(t) / t^{n-1}=-\infty,
$$

a contradiction.

Let $\delta=-1$ and $p_{i}(t)$ be bounded for $i=1, \ldots, l$. Hence $x(t)$ bounded implies $z(t)$ bounded. Integrating the inequality $z^{(n)}(t) \geq f(t)$ for $t \geq t_{1}$ $n$ times successively we get

$$
z(t) \geq \mu_{n}+\mu_{n-1}\left(t-t_{1}\right)+\ldots+\frac{\mu_{1}}{(n-1) !}\left(t-t_{1}\right)^{n-1}+F(t)
$$


for some constants $\mu_{1}, \ldots, \mu_{n}$. Consequently, from the second condition of $\left(\mathrm{A}_{3}\right)$, we obtain

$$
\infty \leq \limsup _{t \rightarrow \infty} z(t) / t^{n-1}=0,
$$

a contradiction.

This completes the proof of the theorem.

Remark 1. Theorem 2(a) generalizes the following result due to Erbe and Zhang [2]: If there exists a function $F(t)$ such that $F^{\prime}(t)=f(t)$, $\liminf \operatorname{in}_{t \rightarrow \infty} F(t)=-\infty$ and $\lim \sup _{t \rightarrow \infty} F(t)=\infty$, then every solution of

$$
[x(t)+p(t) x(t-\tau)]^{\prime}+q(t) y(t-\sigma)=f(t)
$$

oscillates, where $p$ and $q$ are nonnegative continuous functions and $\tau$ and $\sigma$ are positive constants.

Re mark 2. We may note that Theorem 1(a) is applicable to the equation

$$
[x(t)+x(t-\pi)]^{\prime}+x(t-\pi / 2)=\cos t, \quad t \geq \pi,
$$

but it fails to hold true for the equation

$$
[x(t)+x(t-\pi)]^{\prime}+t x(t-\pi / 2)=-t \cos t, \quad t \geq \pi .
$$

On the other hand, Theorem 2(a) cannot be applied to (2.10), but is applicable to (2.11). In particular, $x(t)=-\sin t$ and $x(t)=\sin t$ are oscillatory solutions of (2.10) and (2.11) respectively.

EXAMPLE 2. It is easy to see that all the conditions of Theorem 2(b) are satisfied for

$$
[x(t)+x(t-\pi / 2)]^{\prime}-\left(e^{\pi / 2} \sin t+1+e^{\pi / 2}\right) y(t-\pi / 2)=-e^{t} \sin t,
$$

$t \geq \pi / 2$. Clearly, $x(t)=e^{t}$ is an unbounded nonoscillatory solution of (2.12). Thus the conditions of Theorem 2(b) do not ensure the oscillation of all solutions of (1.2).

Remark 3. Consider the equations

$$
\begin{aligned}
{[x(t)+x(t-\pi)]^{\prime}-x(t-\pi / 2) } & =\cos t, & & t \geq \pi, \\
{[x(t)+2 x(t-\pi)]^{\prime}-t x(t-\pi / 2) } & =(t-1) \cos t, & & t \geq \pi .
\end{aligned}
$$

Clearly, the bounded solutions of (2.13) and (2.14) oscillate by Theorems 1(b) and 2(b) respectively. But Theorem 1(b) fails to hold for (2.14) and Theorem 2(b) cannot be applied to (2.13).

Theorem 3. Assume that

$$
\liminf _{t \rightarrow \infty} \int_{t_{0}}^{t} f(s) d s=-\infty, \quad \limsup _{t \rightarrow \infty} \int_{t_{0}}^{t} f(s) d s=\infty
$$


and

$$
\frac{1}{t^{n-1}} \int_{t_{0}}^{t}(t-s)^{n-1} f_{+}(s) d s \leq \alpha, \quad \frac{1}{t^{n-1}} \int_{t_{0}}^{t}(t-s)^{n-1} f_{-}(s) d s \leq \beta
$$

for $t \geq t_{0}$, where $f_{+}(t)=\max \{f(t), 0\}, f_{-}(t)=\max \{-f(t), 0\}$ and $\alpha>0$ and $\beta>0$ are constants. Then (a) all solutions of (1.2) with $\delta=1$ oscillate, and (b) all bounded solutions of (1.2) with $\delta=-1$ oscillate provided that each $p_{i}(t)$ is bounded.

Proof. Assuming $x(t)$ to be an eventually positive solution of (1.2) and setting $z(t)$ as in (2.8), we obtain (2.9) for $t \geq t_{1}>t_{0}$. If $\delta=1$, then (2.9) gives, for $t \geq t_{1}$,

$$
z^{(n)}(t) \leq f(t),
$$

which on integration yields

$$
z^{(n-1)}(t) \leq z^{(n-1)}\left(t_{1}\right)+\int_{t_{1}}^{t} f(s) d s .
$$

Hence $\liminf \operatorname{in}_{t \rightarrow \infty} z^{(n-1)}(t)=-\infty$. Consequently, for every $L>0$, there exists a $t_{2}>t_{1}$ such that $z^{(n-1)}\left(t_{2}\right)<-L$. We choose $L>\alpha$. Repeated integration of (2.15) from $t_{2}$ to $t$ yields

$$
\begin{aligned}
z(t) & \leq \sum_{k=0}^{n-1} \frac{z^{(k)}\left(t_{2}\right)\left(t-t_{2}\right)^{k}}{k !}+\frac{1}{(n-1) !} \int_{t_{2}}^{t}(t-s)^{n-1} f(s) d s \\
& \leq \sum_{k=0}^{n-1} \frac{z^{(k)}\left(t_{2}\right)\left(t-t_{2}\right)^{k}}{k !}+\frac{1}{(n-1) !} \int_{t_{2}}^{t}(t-s)^{n-1} f_{+}(s) d s
\end{aligned}
$$

which implies that

$$
0 \leq \limsup _{t \rightarrow \infty} \frac{z(t)}{t^{n-1}} \leq \frac{\alpha-L}{(n-1) !}<0,
$$

a contradiction.

Let $\delta=-1$ and $p_{i}(t), i=1, \ldots, l$, be bounded. If $x(t)$ is bounded, then so is $z(t)$. In this case (2.9) implies that

$$
z^{(n)}(t) \geq f(t), \quad t \geq t_{1} .
$$

Integrating (2.16) from $t_{1}$ to $t$ and using the second condition of $\left(\mathrm{A}_{4}\right)$ we have $\lim \sup _{t \rightarrow \infty} z^{(n-1)}(t)=\infty$. So, for every $M>\beta>0$, there exists a 
$t_{3}>t_{1}$ such that $z^{(n-1)}\left(t_{3}\right)>M$. Therefore, from (2.16) we obtain

$$
\begin{aligned}
z(t) & \geq \sum_{k=0}^{n-1} \frac{z^{(k)}\left(t_{3}\right)\left(t-t_{3}\right)^{k}}{k !}+\frac{1}{(n-1) !} \int_{t_{3}}^{t}(t-s)^{n-1} f(s) d s \\
& \geq \sum_{k=0}^{n-1} \frac{z^{(k)}\left(t_{3}\right)\left(t-t_{3}\right)^{k}}{k !}-\frac{1}{(n-1) !} \int_{t_{3}}^{t}(t-s)^{n-1} f_{-}(s) d s
\end{aligned}
$$

which implies that

$$
0 \geq \liminf _{t \rightarrow \infty} \frac{z(t)}{t^{n-1}} \geq \frac{M-\beta}{(n-1) !}>0
$$

a contradiction.

The case $x(t)<0$ may be dealt with similarly, hence the proof of the theorem is complete.

Remark 4. If all the conditions of Theorem 3(a) are satisfied, every solution of

$$
[x(t)+x(t-\pi)]^{\prime \prime}+t x(t-\pi / 2)=-t \cos t, \quad t \geq \pi,
$$

oscillates. Clearly, $x(t)=\sin t$ is such a solution of (2.17). We may note that Theorem 3(a) cannot be applied to equation (2.10), because in this case hypothesis $\left(\mathrm{A}_{4}\right)$ is not satisfied. It also fails to hold for (2.11) since $\int_{t_{0}}^{t} f_{+}(s) d s$ and $\int_{t_{0}}^{t} f_{-}(s) d s$ are not bounded. Further, Theorem 1(a) fails to work for $(2.17)$ as $q(t)=t$ is not $\pi$-periodic and Theorem 2(a) is not applicable to $(2.17)$ as $\left(\mathrm{A}_{3}\right)$ does not hold.

In the following, two results concerning the asymptotic behaviour of solutions of (1.1) are proved.

Theorem 4. Suppose that $n=1, \delta=1, p_{i}(t)$ is bounded, $i=1, \ldots, l$, $q_{k}(t) \geq q>0$ for some $k \in\{1, \ldots, m\}$ and $f(t) \geq 0$ is such that

$$
\int_{t_{0}}^{\infty} s f(s) d s<\infty .
$$

Then all nonoscillatory solutions of (1.1) tend to zero as $t \rightarrow \infty$.

Proof. Let $x(t)$ be a nonoscillatory solution of (1.1). If $x(t)>0$ for $t \geq t_{1}>t_{0}$, then there exists a $t_{2}>t_{1}$ such that $x\left(t-\tau_{i}\right)>0, i=1, \ldots, l$, and $x\left(t-\sigma_{j}\right)>0, j=1, \ldots, m$, for $t \geq t_{2}$. Setting

$$
z(t)=x(t)+\sum_{i=1}^{l} p_{i}(t) x\left(t-\tau_{i}\right)
$$


for $t \geq t_{2}$, we see that $z(t)>0$ and

$$
z^{\prime}(t)=f(t)-\sum_{j=1}^{m} q_{j}(t) x\left(t-\sigma_{j}\right) \leq f(t)-q_{k}(t) x\left(t-\sigma_{k}\right) .
$$

Integration of the above inequality gives

$$
\int_{t_{2}}^{t} x\left(s-\sigma_{k}\right) d s \leq \frac{1}{q}\left[\int_{t_{2}}^{t} f(s) d s+z\left(t_{2}\right)\right],
$$

which, due to (2.18), shows that $x \in L_{1}\left(\left[t_{2}, \infty\right), \mathbb{R}\right)$, the space of Lebesgue measurable functions whose absolute values are integrable. Hence $z \in$ $L_{1}\left(\left[t_{2}, \infty\right), \mathbb{R}\right)$. Thus, $z^{\prime}(t) \leq f(t)$ for $t \geq t_{2}$ implies that

$$
t z(t) \leq t_{2} z\left(t_{2}\right)+\int_{t_{2}}^{t} s f(s) d s+\int_{t_{2}}^{t} z(s) d s \leq \alpha
$$

where $\alpha>0$ is a constant. Consequently, $z(t) \rightarrow 0$ as $t \rightarrow \infty$ and therefore $x(t) \rightarrow 0$ as $t \rightarrow \infty$.

Next let $x(t)<0$ for $t \geq t_{1}>t_{0}$. So $z(t)<0$ and $z^{\prime}(t)>0$ for $t \geq t_{2}$. Hence $-\infty<\lim _{t \rightarrow \infty} z(t) \leq 0$. If $\lim _{t \rightarrow \infty} z(t) \neq 0$, then $z \notin L_{1}\left(\left[t_{2}, \infty\right), \mathbb{R}\right)$. However, from (2.20) we get

$$
\int_{t_{2}}^{t} x\left(s-\sigma_{k}\right) d s \geq \frac{1}{q} z\left(t_{2}\right),
$$

which implies that $x \in L_{1}\left(\left[t_{2}, \infty\right), \mathbb{R}\right)$ and hence $z \in L_{1}\left(\left[t_{2}, \infty\right), \mathbb{R}\right)$, a contradiction. Thus $z(t) \rightarrow 0$ as $t \rightarrow \infty$ and therefore $x(t) \rightarrow 0$ as $t \rightarrow \infty$.

Hence the theorem is proved.

EXAmple 3. By Theorem 4, all nonoscillatory solutions of

$$
\left[x(t)+e^{-t-1} x(t-1)\right]^{\prime}+t e^{-2} x(t-2)=e^{-t}(t-1)-2 e^{-2 t},
$$

$t>3$, tend to zero as $t \rightarrow \infty$. In particular, $x(t)=e^{-t}$ is such a solution.

Theorem 5. Assume that $\delta=1, n=1$, each $p_{i}(t)$ is bounded,

$$
\int_{t_{0}}^{\infty}\left(\sum_{j=1}^{m} q_{j}(t)\right) d t<\infty
$$

and $f(t) \geq 0$. Then all nonoscillatory solutions of (1.1) are unbounded if and only if $\int_{t_{0}}^{\infty} f(t) d t=\infty$.

P r o of. Let $x(t)$ be a nonoscillatory solution of (1.1). Suppose that $x(t)$ is unbounded. Setting $z(t)$ as in (2.19), we see that it is unbounded. Clearly, $x(t)<0$ eventually is not possible, because in this case we have $z(t)<0$ 
and $z^{\prime}(t)>0$ for $t \geq t_{2}>t_{0}$ and hence $z(t)$ is bounded, a contradiction. Thus $x(t)>0$ eventually and hence $z^{\prime}(t)<f(t)$ for $t \geq t_{2}$. Consequently,

$$
z(t)<z\left(t_{2}\right)+\int_{t_{2}}^{t} f(s) d s
$$

which in turn implies, in view of the unboundedness of $z(t)$, that $\int_{t_{2}}^{\infty} f(s) d s=\infty$.

Conversely, suppose that $\int_{t_{2}}^{\infty} f(s) d s=\infty$. If $x(t)$ is bounded, then so is $z(t)$ and there exists a $t_{3}>t_{0}$ such that $\left|x\left(t-\sigma_{j}\right)\right| \leq \beta, j=1, \ldots, m$, for $t \geq t_{3}$. Therefore (2.20) implies that

$$
z^{\prime}(t) \geq f(t)-\sum_{j=1}^{m} q_{j}(t)\left|x\left(t-\sigma_{j}\right)\right|>f(t)-\beta \sum_{j=1}^{m} q_{j}(t) .
$$

Thus, for $t \geq t_{3}$,

$$
z(t)>z\left(t_{3}\right)+\int_{t_{3}}^{t} f(s) d s-\beta \int_{t_{3}}^{t}\left(\sum_{j=1}^{m} q_{j}(s)\right) d s .
$$

Consequently, $z(t) \rightarrow \infty$ as $t \rightarrow \infty$, contradicting the fact that $z(t)$ is bounded.

Hence the theorem is established.

Acknowledgements. The authors are thankful to the referee for his remarks which helped to present the whole paper more elegantly.

\section{References}

[1] Q. Chuanxi and G. Ladas, Oscillations of higher order neutral differential equations with variable coefficients, Math. Nachr. 150 (1991), 15-24.

[2] L. H. Erbe and B. G. Zhang, Oscillation for first order linear differential equations with deviating arguments, Differential Integral Equations 1 (1988), 305-314.

[3] M. K. Grammatikopoulos, G. Ladas and A. Meimardou, Oscillation and asymptotic behaviour of higher order neutral equations with variable coefficients, Chinese Ann. Math. Ser. B 9 (1988), 322-328.

[4] J. Jaros and T. Kusano, On a class of first order nonlinear functional differential equations of neutral type, Czechoslovak Math. J. 40 (1990), 475-490.

[5] P. X. Weng, Oscillation of second order neutral nonlinear functional differential equation, Ann. Differential Equations 7 (1991), 306-315.

[6] L. Wudu, Existence of nonoscillatory solutions of first order nonlinear neutral equations, J. Austral. Math. Soc. Ser. B 32 (1990), 180-192. 
[7] L. Wudu, Oscillations of high order neutral differential equations with oscillatory coefficient, Acta Math. Appl. Sinica 7 (1991), 135-142.

Department of Mathematics

Berhampur University

Berhampur 760 007, India

Reçu par la Rédaction le 11.4.1994

Révisé le 15.9.1994 